\title{
Some topics in the molecular dynamics ensemble
}

\author{
F. Lado \\ Department of Physics, North Carolina State University, Raleigh, North Carolina 27650 \\ (Received 3 August 1981; accepted 13 August 1981)
}

The molecular dynamics method generates averages in an ensemble with constant particle number, volume, energy, and total momentum. For this ensemble, we obtain the exact ideal gas partition function and momentum distribution function, show that a "correction" applied in the past to equation-of-state data of hard core particles is not needed, and rederive a known relationship between potential energy fluctuations and the heat capacity.

\section{INTRODUCTION}

By numerically solving the Newtonian equations of motion stepwise in time, a molecular dynamics (MD) calculation generates the explicit microscopic time evolution of a model many-body system. ${ }^{1}$ Equilibrium properties of the system (as well as dynamical ones) can then be obtained as time averages over this recorded history. It is the basic postulate of statistical mechanics that such time averages at equilibrium can be reproduced by appropriate ensemble averages, disregarding the temporal sequence that microstates can take on. Since the energy is a constant of the motion, the MD method must be matched to a microcanonical ensemble. However, the additional constraint of a fixed center-of-mass momentum which follows from Newton's equations distinguishes this microcanonical ensemble from the usual one; Wood $^{2}$ has called it the molecular dynamics ensemble.

This note examines three loosely linked topics in the molecular dynamics ensemble. (1) The exact partition function and momentum distribution function are obtained for an ideal gas in this ensemble. (2) A "correction" applied to data from hard core fluids ${ }^{3}$ to permit comparison with Monte Carlo results is shown to be unnecessary. (3) An expression for the fluctuation of the kinetic or potential energy obtained by Lebowitz et al. ${ }^{4}$ by transforming canonical ensemble quantities is rederived entirely within the $\mathrm{MD}$ ensemble.

\section{THE IDEAL GAS}

The partition function of an ideal gas of $N$ molecules in a volume $V$ in the usual microcanonical ensemble,

$$
Z_{N V B}^{(0)}=V^{N} \int d \mathrm{p}^{N} \delta\left(E-\sum_{j=1}^{N} p_{j}^{2} / 2 m\right)
$$

is proportional to the surface area of a $\nu N$-dimensional sphere of radius $(2 m E)^{1 / 2}$, where $E$ is the thermal kinetic energy of the gas, $m$ the molecular mass, and $\nu=1,2$, or 3 the spatial dimension. It is most easily evaluated by first determining the volume of the sphere. The result is ${ }^{5}$

$$
Z_{N V E}^{(0)}=2 \pi m V^{N}(2 \pi m E)^{\nu N / 2-1} / \Gamma(\nu N / 2),
$$

with $\Gamma(x)$ the gamma function. When the second constraint is added in the $\mathrm{MD}$ ensemble, the partition function for total energy $E$ and center-of-mass momentum $\mathbf{P}$ becomes

$$
Z_{N V E P}^{(0)}=V^{N} \int d \mathrm{p}^{N} \delta\left(E-\sum_{j=1}^{N} p_{j}^{2} / 2 m\right) \delta\left(\mathrm{P}-\sum_{j=1}^{N} \mathrm{p}_{j}\right),
$$

and is proportional to the intersection of a sphere and a plane in the $\nu N$-dimensional momentum space. This integral is evaluated in the Appendix by Fourier-expanding the Dirac delta functions to permit integration over the $p_{j}$ separately. The result is

$$
\begin{array}{r}
Z_{N V B P}^{(0)}=\frac{2 \pi m V^{N}\left[2 \pi m\left(E-P^{2} / 2 N m\right)\right]^{\nu(N-1) / 2-1}}{N^{\nu / 2} \Gamma[\nu(N-1) / 2]}, \\
E \geq P^{2} / 2 N m .
\end{array}
$$

Besides the obvious generalization of the thermal energy to total energy $E$ less the mechanical energy of the center-of-mass motion, the new constraint has caused an effective reduction in the number of molecules from $N$ in Eq. (2) to $N-1$ in Eq. (4); the thermal motion of one molecule has been "frozen out." This leads to a slightly higher temperature for the same thermal energy $[\beta$ $\left.\equiv\left(k_{B} T\right)^{-1}\right]$

$$
\beta_{N V E P}=\frac{\partial \ln Z_{N V E R}}{\partial E}=\frac{\nu(N-1) / 2-1}{E-P^{2} / 2 N m},
$$

but unchanged equation of state

$$
(\beta p)_{N V E P}=\theta \ln Z_{N V E P} / \theta V=N / V \text {. }
$$

The intensive variable conjugate to $P$ is defined by

$$
\begin{aligned}
\mathrm{b}_{N V E P} & =\nabla_{P} \ln Z_{N V E P} \\
& =-\beta_{N V E P} \mathbf{P} / N m .
\end{aligned}
$$

Thus, $b=-\beta u$, where $u$ is the mean molecular velocity in the gas.

The momentum distribution function is defined by

$$
f(\mathrm{p})_{N V E P}=N^{-1}\left\langle\sum_{j=1}^{N} \delta\left(\mathrm{p}-\mathrm{p}_{j}\right)\right\rangle_{N V E P},
$$

where the angular brackets denote an ensemble average. Evaluation of Eq. (6) proceeds much as before with Eq. (3), described in the Appendix. The result is

$$
\begin{aligned}
f(\mathrm{p})_{N V E P}= & \left(\frac{N}{N-1}\right)^{\nu / 2} \frac{\Gamma[\nu(N-1) / 2]}{\Gamma[\nu(N-2) / 2]} \\
& \times \frac{\left[1-\frac{N}{N-1} \frac{(\mathrm{p}-\mathrm{p} / N)^{2}}{2 m E-P^{2} / N}\right]^{\nu(N-2) / 2-1}}{\left[2 \pi m\left(E-P^{2} / 2 N m\right)\right]^{\nu / 2}},
\end{aligned}
$$

where the bracketed function of $p$ must be non-negative, i.e., 


$$
\frac{(\mathrm{p}-\mathrm{P} / N)^{2}}{2 m} \leq \frac{N-1}{N}\left(E-\frac{p^{2}}{2 N m}\right)
$$

The mean momentum

$$
\langle\mathrm{p}\rangle_{N V E P}=\mathrm{P} / N
$$

is obtained from Eq. (7) by inspection and the variance by calculation, whence the molecular fluctuation energy

$$
\frac{(\Delta P)_{N Y E P}^{2}}{2 m}=\frac{\left\langle\left(\mathrm{p}-\langle\mathrm{p}\rangle_{N V E P}\right)^{2}\right\rangle_{N V E P}}{2 m}=\frac{E-P^{2} / 2 N m}{N}
$$

is seen to be just the mean thermal energy per molecule, as expected.

Just as the more familiar canonical ensemble partition function can be obtained by Laplace transformation from the microcanonical ensemble, ${ }^{B}$

$$
\begin{aligned}
Z_{N V B}^{(0)} & =\int_{0}^{\infty} d E e^{-\beta E} Z_{N V E}^{(0)} \\
& =V^{N}(2 \pi m / \beta)^{\nu_{N} / 2}
\end{aligned}
$$

so too can a generalized canonical ensemble be constructed from the MD ensemble. We lift both the constraints on $E$ and $\mathbf{P}$ to get

$$
\begin{aligned}
Z_{N V B b}^{(0)} & =\int d \mathrm{P} \int_{P^{2} / 2 N m}^{\infty} d E \exp (-\beta E-\mathrm{b} \cdot \mathrm{P}) Z_{N V E P}^{(0)} \\
& =V^{N}(2 \pi m / \beta)^{\nu^{N} / 2} \exp \left(N m b^{2} / 2 \beta\right)
\end{aligned}
$$

as first obtained by Grad, ${ }^{7}$ where now $\beta$ and $b$ are the independent variables and the dependent ones are

$$
\begin{aligned}
& E_{N V B b}=-\theta \ln Z_{N V B b} / \theta \beta=\nu N / 2 \beta+N m b^{2} / 2 \beta^{2}, \\
& \beta P_{N V B b}=\theta \ln Z_{N V B b} / \theta V=N / V, \\
& \mathbf{P}_{N V B b}=-\nabla_{b} \ln Z_{N V B b}=-N m b / \beta .
\end{aligned}
$$

A similar transformation of Eq. (7) yields a far simpler distribution function,

$$
\begin{aligned}
f(\mathrm{p})_{N V B b} & =Z_{N V B b}^{-1} \int d \mathrm{P} \int d E \exp (-\beta E-\mathrm{b} \cdot \mathrm{P}){Z_{N V B P}^{(0)} f(\mathrm{p})_{N V E P}} \\
& =(2 \pi m / \beta)^{-\nu / 2} \exp \left[-\beta(\mathrm{p}+m \mathrm{~b} / \beta)^{2} / 2 m\right] .
\end{aligned}
$$

When the same system is to be studied in two different ensembles, the independent parameters must be linked. Thus, one can choose a temperature and mean velocity $(\beta$ and $b)$ in the generalized canonical ensemble and obtain the averages $E_{N V B b}$ and $\mathrm{P}_{N V \beta B}$; the independent $E$ and $P$ variables of the $M D$ ensembles are then chosen to match these canonical results,

$$
E=E_{N V \beta b}, \quad \mathrm{P}=\mathrm{P}_{N V \beta b} .
$$

It follows then from Eqs. (5) and (12), that the MD temperature is slightly higher,

$$
\frac{\beta_{\text {NVRE }}}{\beta}=\frac{N-5 / 3}{N} \text {, }
$$

as is consequently the pressure, since

$$
(\beta p)_{N V B b}=(\beta p)_{N V E P},
$$

while the mean velocity $u=-b / \beta$ is the same,

$$
\frac{b}{\beta}=\frac{b_{N V E R}}{\beta_{N V E P}} \text {. }
$$

It will be noted, that the generalized canonical ensemble [Eq. (11)] reduces to the usual canonical ensemble when $b=0$ (i.e., $u=0$ ), while the MD ensemble still differs from the microcanonical for $\mathbf{P}=0$. Since $\mathbf{u}=\mathbf{0}$ always holds in the canonical ensemble, it appears that its proper microcanonical conjugate in all cases is precisely the $\mathrm{MD}$ ensemble with $\mathbf{P}=0$, and not the usual microcanonical ensemble with unconstrained momentum.

\section{INTERACTING SYSTEMS}

For real systems with a configuration-dependent potential energy $U\left(\mathbf{r}^{N}\right)$, we have

$Z_{N V B P}=\int d \mathbf{r}^{N} d \mathbf{p}^{N} \delta\left[E-U\left(\mathbf{r}^{N}\right)-\Sigma p_{j}^{2} / 2 m\right] \delta\left(\mathbf{P}-\Sigma \mathbf{p}_{j}\right)$,

and, in general, the two sets of integrations over $r^{N}$ and $\mathrm{p}^{N}$ cannot be separated. The single exception to this rule occurs with the hard-core interaction, for which $U\left(\mathbf{r}^{N}\right)$ is either $\infty$ if cores intersect each other or 0 if they do not. Then Eq. (16) becomes

$$
\begin{aligned}
Z_{N V E P} & =\int d \mathbf{r}^{N} d \mathbf{p}^{N} \delta\left(E-\Sigma p_{j}^{2} / 2 m\right) \delta\left(\mathbf{P}-\Sigma p_{j}\right) W\left(\mathbf{r}^{N}\right) \\
& =Z_{N V E P}^{001} \Omega_{N V},
\end{aligned}
$$

where

$$
\begin{aligned}
\Omega_{N V} & =V^{-N} \int d r^{N} W\left(r^{N}\right), \\
W\left(r^{N}\right)=0, & U\left(\mathbf{r}^{N}\right)=\infty, \\
& =1, \quad U\left(\mathbf{r}^{N}\right)=0 .
\end{aligned}
$$

The temperature and mean velocity are those of the ideal gas [Eq. (5)], while the pressure is given by

$$
(\beta p)_{N V E P}=N / V+\theta \ln \Omega_{N V} / \theta V .
$$

Of course, the canonical partition function is always separable in the classical limit and so we have immediately

$Z_{N V B b}=Z_{N V B b}^{(0)} V^{-N} \int d r^{N} \exp \left[-\beta U\left(r^{N}\right)\right]=Z_{N V B b}^{(0)} \Omega_{N V}$,

with the same configuration integral $\Omega_{N V}$. Hence, we find

$$
(\beta p)_{N V B D}=N / V+\theta \ln \Omega_{N V} / \partial V=(\beta p)_{N V E P} .
$$

Assuming the basic postulate of equilibrium statistical mechanics, Monte Carlo calculations of the left-hand side and $\mathrm{MD}$ calculations of the right-hand side should thus yield the same equation of state without corrections. Small discrepancies in the early hard disk data ${ }^{3}$ cannot be due to the difference in ensembles.

For potentials other than hard core $U\left(r^{N}\right)$ will take on a range of values and the separation of Eq. (17) cannot be made. Instead, using an obvious change in notation better suited to the present purpose, we write Eq. (16) in the form

$$
\begin{aligned}
Z(E)= & \int d U \int d \mathrm{r}^{N} \delta\left[U-U\left(\boldsymbol{r}^{N}\right)\right] \\
& \times \int d \mathrm{p}^{N} \delta\left(E-U-\Sigma p_{j}^{2} / 2 m\right) \delta\left(\mathrm{P}-\Sigma \mathrm{p}_{j}\right) \\
& =\int d U Z_{0}(E-U) Z_{c}(U),
\end{aligned}
$$


where $Z_{0}$ is the ideal gas partition function and

$$
Z_{c}(U)=V^{-N} \int d \mathbf{r}^{N} \delta\left[U-U\left(\boldsymbol{r}^{N}\right)\right],
$$

that of a configuration-space microcanonical ensemble. Equation (23) describes two thermally interacting subsystems, with fluctuating energy $U$. Since, for large $N, Z_{0}(E-U)$ is a rapidly decreasing and $Z_{c}(U)$ a rapidly increasing function of $U$, their product will be narrowly distributed about the peak at some $U=\hat{U}$ and we may use standard asymptotic techniques to evaluate the integral. Expanding the logarithm of the integrand about $U=\hat{U}$, we have

$$
\begin{aligned}
\ln Z_{0}(E-U) & +\ln Z_{c}(U)=\ln Z_{0}(E-\hat{U})+\ln Z_{c}(\hat{U}) \\
+ & {\left[-\beta_{0}(E-\hat{U})+\beta_{c}(\hat{U})\right](U-\hat{U}) } \\
+ & \frac{1}{2}\left[\beta_{0}^{\prime}(E-\hat{U})+\beta_{c}^{\prime}(\hat{U})\right](U-\hat{U})^{2}+\cdots,
\end{aligned}
$$

where,

$$
\beta_{0, c}(E) \equiv \partial \ln Z_{0, c}(E) / \theta E
$$

are the inverse temperatures of the momentum and configuration space subsystems. Equilibrium occurs for

$$
\beta_{0}(E-\hat{U})=\beta_{c}(\hat{U})=\beta(E),
$$

which defines $\hat{U}_{\text {; }}$ with heat capacity $C$ defined by

$$
\beta^{\prime}(E)=-k_{B} \beta^{2}(E) / C,
$$

we have the usual Gaussian integrand for $\mathrm{Eq}$. (23),

$$
\begin{aligned}
Z_{0}(E-U) Z_{c}(U) \simeq & Z_{0}(E-\hat{U}) Z_{c}(\hat{U}) \\
& \times \exp \left\{-\frac{1}{2} k_{B} \beta^{2}\left(1 / C_{0}+1 / C_{c}\right)(U-\hat{U})^{2}\right\},
\end{aligned}
$$

from which the variance of $U$ (or equivalently of $K=E$ $-U$ ) emerges by inspection as

$$
\begin{aligned}
\sigma_{U}^{2} & =\left[k_{B} \beta^{2}\left(\frac{1}{C_{0}}+\frac{1}{C_{c}}\right)\right]^{-1} \\
& =\frac{C_{0}}{k_{B} \beta^{2}}\left(1-\frac{C_{0}}{C}\right)=\sigma_{K}^{2} .
\end{aligned}
$$

Here, $C_{0}$ and $C_{c}$ are the respective heat capacities of the momentum and configuration degrees of freedom and $C=C_{0}+C_{c}$ that of the whole system. Equation (30) was first obtained by Lebowitz et al. " by a transformation from canonical ensemble fluctuations.

\section{APPENDIX: EVALUATION OF THE INTEGRAL FOR $Z_{\text {NVEP }}$}

The Dirac delta function has the Fourier expansion

$$
\delta(x)=\frac{1}{2 \pi} \int_{-\infty}^{\infty} d k e^{i k x}=\lim _{\epsilon-0^{+}} \frac{1}{2 \pi i} \int_{\epsilon-i \infty}^{\epsilon+i \infty} d z e^{2 x},
$$

where the second form will be useful below in dealing with a singularity. Thus, we may write (leaving the limit $\epsilon \rightarrow 0^{+}$implied)

$$
\begin{aligned}
I \equiv & \int d \mathrm{p}^{N} \delta\left(A-\sum_{j=1}^{N} p_{j}^{2}\right) \delta\left(\mathrm{B}-\sum_{j=1}^{N} \mathrm{p}_{j}\right) \\
= & \int_{C} \frac{d z}{2 \pi i} \int \frac{d \mathbf{k}}{(2 \pi)^{\nu}} \exp (z A+i \mathrm{k} \cdot \mathrm{B}) \\
& \times\left[\int d \mathrm{p} \exp \left(-z p^{2}-i \mathrm{k} \cdot \mathrm{p}\right)\right]^{N} \\
= & \int_{C} \frac{d z}{2 \pi i} \exp (z A)\left(\frac{z}{\pi}\right)^{-\nu N / 2} \int \frac{d \mathrm{k}}{(2 \pi)^{\nu}} \\
& \times \exp \left(-N k^{2} / 4 z+i \mathrm{k} \cdot \mathrm{B}\right) \\
= & \frac{\pi^{\nu(N-1) / 2}}{N^{\nu / 2}} \int_{C} \frac{d z}{2 \pi i} \exp \left[z\left(A-B^{2} / N\right)\right](z)^{-\nu(N-1) / 2},
\end{aligned}
$$

where the contour $C$ is that in Eq. (A1) and the limits on all other integrals are $-\infty$ to $+\infty$. If $A-B^{2} / N$ is negative, the contour in (A2) can be closed with a halfcircle in the right-half plane, excluding the singularity; the integral vanishes and so

$$
I=0 \text {, if } A-B^{2} / N<0 \text {. }
$$

If $A-B^{2} / N$ is positive, on the other hand, the halfcircle can be drawn in the left-hand plane, enclosing the singularity; the contour integral then yields ${ }^{8}$

$$
I=\pi^{\nu(N-1) / 2}\left(A-B^{2} / N\right)^{\nu(N-1) / 2-1} / \Gamma[\nu(N-1) / 2]
$$

$$
\text { if } A-B^{2} / N>0 \text {. }
$$

${ }^{1}$ B. J. Alder and T. E. Wainwright, J. Chem. Phys. 31, 459 (1959); A. Rahman, Phys. Rev. A 136, 405 (1964).

${ }^{2}$ W. W. Wood, in Fundamental Problems in Statistical Mechanics, edited by E. G. D. Cohen (North-Holland, New York, 1975).

${ }^{3}$ W. G. Hoover and B. J. Alder, J. Chem. Phys. 46, 686 (1967).

${ }^{4}$ J. L. Lebowitz, J. K. Percus, and L. Verlet, Phys. Rev. 153,250 (1967).

${ }^{5}$ See, for example, K. Huang, Statistical Mechanics (Wiley, New York, 1963), p. 152.

${ }^{6} \mathrm{See}$, for example, R. Kubo, Statistical Mechanics (NorthHolland, New York, 1971), p. 22.

${ }^{7}$ H. Grad, Comm. Pure and Appl. Math. 5, 455 (1952).

${ }^{8}$ Handbook of Mathematical Functions, edited by M. Abramowitz and I. A. Stegun (U.S.GPO, Washington, DC, 1964), p. 255. 